\title{
Laparoscopic Splenectomy- Feasible With Better Outcomes in Hematological Conditions - An Observational Study
}

\author{
Srinivasan Ulagendraperumal ${ }^{1}$, T P R Bharadwaj ${ }^{2}$, Naganath Babu Obla ${ }^{3}$ \\ ${ }^{1}$.Deparment of surgical gastroenterology, Aysha hospital 91, Millers Road, Kilpauk, Chennai, India. \\ ${ }^{2}$ Deparment of Hematology, Aysha hospital 91, Millers Road, Kilpauk, Chennai, India. \\ ${ }^{3}$ Department of surgical gastroenterology, Govt. Royapettah hospital, Royapettah, Chennai, India.
}

\section{Introduction}

Laparoscopic splenectomy was first reported in the year 1991 by French surgeons Delaitre and Maignien. Since then it has been recognized as an accepted procedure for elective splenectomies in the management of various hematological as well as neoplastic conditions [1,2]. Though, many major reports were available in the international arena, not much was reported from the southern part of India. We are reporting a case series of laparoscopic splenectomies performed by a single laparoscopic surgeon over a period of 2 years with a minimum follow-up of 2 months and a maximum follow-up of 1year and 10 months. One of the major observations in this series was its better outcome in terms of operative time and intraoperative blood loss.

\section{Methods}

Retrospective review of all patients who had undergone elective splenectomies between April 2012 and May 2014 were included. All laparoscopic splenectomies were performed by a single surgeon (first author). All the patients were fully evaluated and referred for surgery by our consultant hematologist. Evaluation includes ultrasound scan abdomen to assess spleen size, complete blood counts including platelet count. Intravenous hydrocortisone was administered on a routine basis for all the patients with ITP. Preoperative platelet counts were optimized by our hematologist in all but 3 patients who had steroid refractory ITP. These 3 patients had undergone surgery with a platelet count between 10000 and 20000 as quasi-emergency. All patients received pneumococcal, meningococcal and Hemophilus influenza type B vaccines 2 weeks before surgery.

\section{Operative Procedure}

14 patients with normal size to moderate splenomegaly were operated in right lateral position using four ports. 1 patient with hereditary spherocytosis with spleen size of $21 \times 12 \times 10 \mathrm{~cm}$ was operated in supine position using four ports.

Pneumoperitoneum was created using Veress needle through the Palmer's point in all the patients with normal sized spleens. For patients with moderate and severe splenomegaly $(18 \mathrm{~cm}$ and $21 \mathrm{~cm}$ maximum dimension respectively) pneumoperitoneum was created through the umbilicus. Pressure was kept at $14 \mathrm{~mm} \mathrm{Hg}$. Port placement was done as following: $12 \mathrm{~mm}$ port at the Palmer's point in the mid-clavicular line, $10 \mathrm{~mm}$ port just to the left of the midline above the level of the $12 \mathrm{~mm}$ port, $5 \mathrm{~mm}$ port laterally in the anterior axillary line at the level of the $12 \mathrm{~mm}$ port, and a $5 \mathrm{~mm}$ port just below the level of the xiphisternum.

Splenic flexure was mobilized down and all the splenic attachments like spleno-renal, spleno-colic and gastro-splenic were divided using vessel sealing device [Fig. 1]. Attachments around the splenic hilum were dissected and a posterior plane created in the region of the hilum of spleen behind the tail of the pancreas using a laparoscopic 'gold finger' instrument to enable insertion of endo stapler which was used in initial 12 patients to divide the splenic pedicle [Fig. 2,3]. In the last three ITP patients we used $10 \mathrm{~mm}$ vessel sealing device to divide the splenic hilar vessels. After this the phrenic ligament was divided using diathermy hook.

Specimens were retrieved using custom made plastic endobags through the $12 \mathrm{~mm}$ port by morcellation using sponge holding forceps. In the 2 patients with severe splenomegaly an incision of about $5 \mathrm{~cm}$ was made by joining the two lateral most ports to retrieve the specimen. A $14 \mathrm{~F}$ suction catheter was left insitu as tube drain in first 12 patients in this series. Drains were removed on the second post operative day in all the patients.

Post operative platelet counts were obtained on the first post operative day and repeated daily till the time of discharge for all the patients.

Demographic data recorded were age and sex of the patients. Clinical data recorded includes indications for surgery, combined surgical procedures, duration of surgery, intra operative and post operative events and complications, need for blood and product transfusions, postoperative recovery, morbidity and mortality. 


\section{Results}

Fifteen patients who had undergone laparoscopic splenectomy (LS) between April 2012 and May 2014 were retrospectively analyzed. Of these $60 \%$ patients were females. The age ranged from 15 years to 75 years and the mean age was 37 years. The most common indication was ITP (eleven patients). Of these 6 surgeries were done for complications of steroid and 5 were done for refractory ITPs (refractory to steroids and immunosuppressants). Other indications include hereditary spherocytosis, autoimmune hemolytic anemia and splenectomy as biopsy for lymphoproliferative disorders. The mean preoperative platelet count in ITP patients was 48700 (range 10000 to 130000). 13 patients had normal to mildly enlarged spleens and 2 patients had moderate to severe splenomegaly. These two patients received open incisions for retrieval of specimens apart from one patient who was opened for an intraoperative bleeding control. 11 patients had BMI of less than 30, two patients had BMI between 30 and 40 and two had a BMI above 40. One patient had a simultaneous umbilical hernia repair by open method along with LS (on-lay mesh repair). The mean operating time was 74 minutes (range 45min to 90min) in LS alone cases; $130 \mathrm{~min}$ in patient with combined umbilical repair. The overall mean estimated blood loss was $159.3 \mathrm{ml}$ (range: $50 \mathrm{ml}$ to $500 \mathrm{ml}$ ). Excluding the patient with an uncontrollable bleed and a patient with combined umbilical hernia repair it becomes drastically reduced to $114.6 \mathrm{ml}$. The patient in whom there was an uncontrollable bleed from the pancreatic tail region, the blood loss was $500 \mathrm{ml}$ which necessitated opening by a $10 \mathrm{~cm}$ incision in the left hypochondrium. Only two patients received blood transfusion intraoperatively, one was the patient with an uncontrollable bleed mentioned above and the other was the patient with hereditary spherocytosis who had extensive peri-splenic adhesions and low preoperative hemoglobin. Two ITP patients with preoperative platelet counts of 10000 received preoperative platelet transfusion, 2 units each during the initial phase of this case series. Later on none of our patients were given blood or blood products.

The specimen was removed through a small incision of around $5 \mathrm{~cm}$ made by joining the lateral-most ports in two patients with moderate to severe splenomegaly. None of the patients in this series had accessory spleen.

The mean hospital stay was 4.83 days (range: 3 to12 days). Postoperative complications occurred in 6 patients, wound sepsis in 3; septic collection of around $6 \mathrm{ml}$ in the region of the spleen in 1, left-sided pleural effusion in 1 and the patient with mesh repair for umbilical hernia had excessive seroma that lasted for 2 months. This patient stayed in the ward for 12 days and got discharged with the suction drain which was removed on the 19th POD. After removal of the drain tube this patient continued to have seroma which lasted for 2 months before resolving. All the patients were followed up for minimum of 2 months. All the ITP patients except one had a very good response pertaining to the improvement in platelet count. A 40 year old male with refractory ITP had a rise to a lifetime maximum of 40000, (preoperative value was 10000) and continued to maintain at that level during follow-up.

Restoration of oral feeding was done on the first post operative day for all except three patients with ITP with complications of long term steroids, who developed prolonged ileus. Oral feeding was started on the 3rd postoperative day in these patients. Mortality was nil in this series.

\section{Discussion}

Although many of the hospitals in this part of the world, (south India), performs advanced laparoscopic procedures including laparoscopic splenectomies, to our knowledge, a series of LS by a single surgeon has not been published. Due to the presence of a Hematologist in our hospital we use to get many referrals for hematological conditions.

As in any other laparoscopic procedures, LS also is associated with less postoperative pain and less hospital stay which is translated into less costs ${ }^{[3]}$. In our hospital, splenectomy was being done from 1986 and all the procedures were open splenectomies before 2012. The most common indication for splenectomy in this series was ITP, accounting for about $73 \%$ of the cases. This is comparable to the international data ${ }^{[4]}$. A severe splenomegaly was defined as one with a maximum dimension of $20 \mathrm{~cm}^{[5]}$. Two cases with moderate to severe splenomegaly, an autoimmune hemolytic anemia and a hereditary spherocytosis were operated in this study.

The overall complication rate in this study was $40 \%$. All the complications were minor complications like wound sepsis, minimal pleural effusion and minimal intra-abdominal collection. The entire wound related complications occurred in those patients who required an incision to retrieve the spleen. Wound sepsis and pleural effusion were managed conservatively. Intra-abdominal collection was aspirated once and sent for fluid culture sensitivity, which grew E.coli sensitive to Amikacin. Most of the postoperative morbidity in these patients seems to be as a result of the immunocompromized state and the use of steroids. Adequate optimization of the patients before surgery and strict aseptic measures in the postoperative period is essential to minimize the morbidity. Return to work was relatively early in most of our patients in view of the laparoscopic approach.

Operative times, indirectly translating into less time under anaesthesia and less hospital stay are the factors that determine the efficacy of any surgical procedure. The mean operative time in this study was 74 
minutes which becomes reduced to 65.3 minutes, if the sole patient with combined umbilical hernia surgery was not included. This low mean operative time was considerably lesser than that of other studies which reported mean operative times in the range of 88 minutes to 188 minutes ${ }^{[6]}$. Comparing the international studies on open splenectomy, LS has a longer operative time ${ }^{[7]}$. A study by Rescorla et al describes an average operating time of 115 minutes for LS compared to 83 minutes for OS, which in any case longer than the operating time for LS in this study ${ }^{[3]}$. Several factors like BMI, previous surgery, splenic size and surgeon's experience, might influence the operative time. The reduction in operative time in this study may be attributed to the use of vessel sealing device, in addition to the small splenic size in most of the patients (range 60gm-160gm in 13 patients; 900gm and $1550 \mathrm{gm}$ in two patients respectively) and only few patients with high BMI. All the surgeries were performed by a single well experienced senior laparoscopic surgeon.

Only one patient was converted (overall conversion rate was $0.15 \%$ ) for an uncontrollable bleed which is attributed to the failure of the energy device and diffuse nature of the bleed in the tail of the pancreas. Bleeding from splenic hilum and bleeding due to enlarged lymph nodes in the splenic hilar region were reported as causes for conversion to open in few studies ${ }^{[8,9]}$.

None of the patients in this series had relaparotomies as a result of postoperative bleeding as it was reported in few studies ${ }^{[2]}$. The average length of stay was comparable with other studies, excluding the one patient with combined umbilical hernia repair Though we have used drains liberally for the first 12 cases, we stopped using drains for the rest of our patients in whom we were stringent in avoidance of injury to the stomach and pancreas as well as achieving hemostasis.

Though it was said that, there is significant risk of bleeding due to spleen's rich vascularity and intimate relationship with adjacent organs, LS could be carried out with less blood loss if care is taken to the anatomical details and usage of modern equipment. The average blood loss in this series of patients was $159 \mathrm{ml}$ which is comparatively lesser than other published studies ${ }^{[10]}$. As reported by Dan et al, it should be admitted that the bleeding that led to the conversion in this series was surgical and not directly due to thrombocytopenia ${ }^{[6]}$. The bleeding occurred after the spleen had been dissected out which warranted an opening for control. The cause of uncontrollable bleeding from the tail of the pancreas was attributed to the failure of the energy device which reiterates the need for a backup to prevent these events. This is one of the two patients who required blood transfusions in this series. The other case was a severe splenomegaly with peri-splenic adhesions due to hereditary spherocytosis with anemia. The need for meticulous dissection and the availability of appropriate equipment could not be overemphasized for the performance of successful and event-free LS.

In conclusion LS could be performed safely in carefully selected patients, with adequate optimization, especially by a hematologist for hematological indications, if carried out by a senior laparoscopic surgeon even in a small setting with limited resources.

\section{References}

[1]. Delaitre B1, Champault G, Barrat C, Gossot D, Bresler L, Meyer C, Collet D, Samama G.Laparoscopic splenectomy for hematologic diseases. Study of 275 cases. French Society of Laparoscopic Surgery. Ann Chir. 2000 Jul;125(6):522-9.

[2]. Makrin V1, Avital S, White I, Sagie B, Szold A.Laparoscopic splenectomy for solitary splenic tumors.Surg Endosc. 2008Sep;22(9):2009-12. doi: 10.1007/s00464-008-0024-8. Epub 2008 Jul 2.

[3]. Rescorla FJ, Breitfeld PP, West KW, et al. A case controlled comparison of open and laparoscopic splenectomy in children.Surgery. 1998; $124: 670-675$.

[4]. Park AE, Birgisson G, Mastrangelo MJ, et al.Laparoscopic splenectomy: outcomes and lessons learned from over 200 cases. Surgery. 2000;128:660-667.

[5]. Poulin, EC and Thibault, C. The anatomical basisfor laparoscopic splenectomy.J Canadien De Chirurgie. 1993; 36: 484-488.

[6]. Dilip Dan, FACS, Dave Harnanan, MBBS, Kirk Gooden, MBBS, Shiva Seetahal, MBBS,Seetharaman Hariharan, MD, Vijay Naraynsingh, FRCSLaparoscopic Splenectomy in a Minimal Resource Setting -A Case Series From the CaribbeanSurg Laparosc Endosc Percutan Tech 2010;20:e125-e128

[7]. Sapucahy Manuela V. ; Faintuch Joel ; Bresciani Cláudio J.C. ; Bertevello Pedro L. ; Habr-Gama Angelita ; Gama-Rodrigues Joaquim José. Laparoscopic versus open splenectomy in the management of hematologic disease.Revista do Hospital das Clínicas. 2003; 58(5):243-249.

[8]. Emmermann A1, Zornig C, Peiper M, Weh HJ, Broelsch CELaparoscopic splenectomy. Technique and results in a series of 27 cases.Surg Endosc. 1995 Aug;9(8):924-7.

[9]. Kiudelis M1, Endzinas Z, Mickevicius A, Reckauskas M.Introduction of laparoscopic splenectomy for the treatment of immune thrombocytopenic purpura.Zentralbl Chir. 2004 Apr;129(2):108-13.

[10]. Chowbey PK1, Goel A, Panse R, Sharma A, Khullar R, Soni V, Baijal M.Laparoscopic splenectomy for hematologic disorders: experience with the first fifty patients.J Laparoendosc Adv Surg Tech A. 2005 Feb;15(1):28-32. 
Laparoscopic splenectomy- Feasible with better outcomes in hematological conditions ....

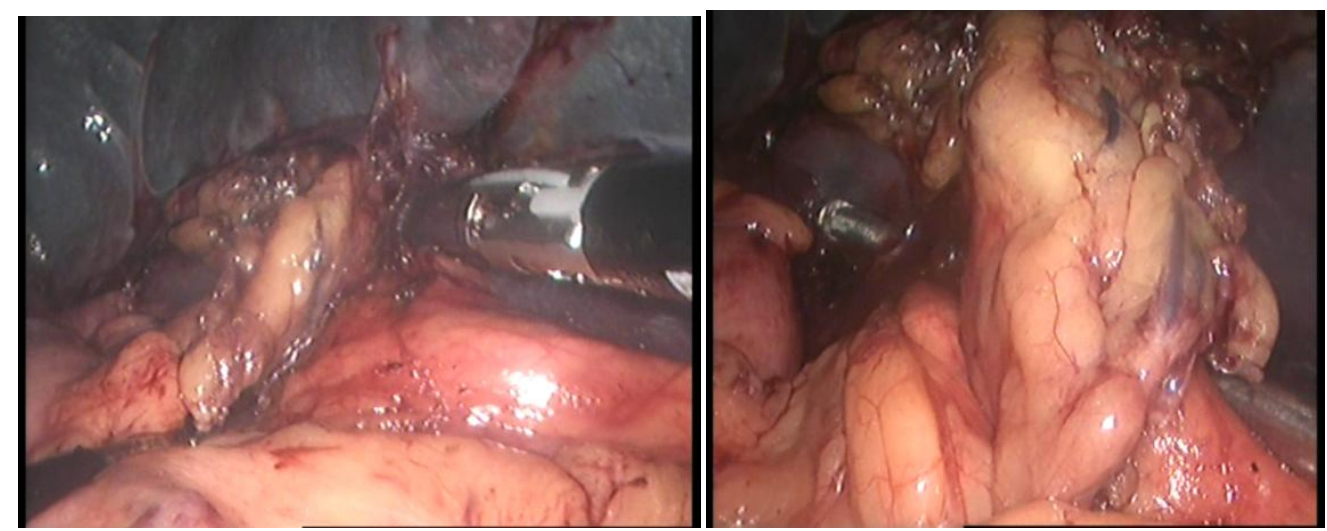

Figure 1. Ligasure being used to divide posterior to the tail of pancreas

Figure 2. Gold finger instrument introduced hilar vessels

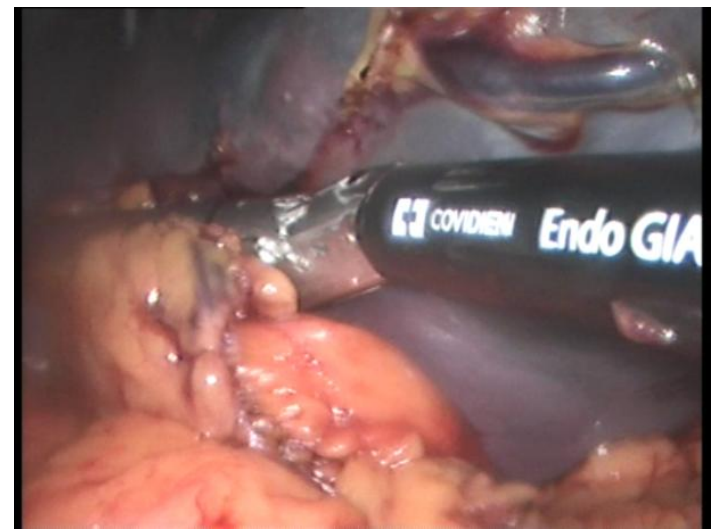

Figure 3. Endoscopic vascular stapler to divide splenic pedicle 